\title{
Study on Silk/Carbamate Films
}

\author{
Hua Fu ${ }^{a}$, Zhu-ping Yin ${ }^{b}$, Feng $W^{c}{ }^{c}$, Kai Liu ${ }^{d}$, Shen-zhou $L^{f^{*}}$ \\ Engineering Laboratory for Modern Silk, College of Textile and Clothing Engineering, Soochow \\ University, Suzhou, China, 215123 \\ flushenzhou@suda.edu.cn \\ *Corresponding author
}

Keywords: Silk Fibroin, Carbamate, Blend Film, Cytocompatibility, Cornea Stroma Regeneration.

\begin{abstract}
In this work, silk fibroin/carbamate blend films are fabricated in different ratios to improve the appropriate properties required for corneal stroma cell regeneration on the films. The structure of the films, the moisture content, protein dissolution loss rate, mechanical properties and light transmittance are investigated. The cytocompatibility of the films is tested with keratinocytes. The result shows that silk II crystalline structure in the film increases with the increase of carbamate content in the film. The light transmittance of the films is above $85 \%$. The light transmittance at wet state is increased when the films are dipped in deionized water at room temperature for 24 hours. The tensile strength and elongation at break of the films are high enough for regeneration of corneal stroma cells both in dry and wet states. The blended films are able to support adhesion and proliferation of keratinocytes. This fibroin-carbamate blend films may offer an option for the corneal tissue engineering application.
\end{abstract}

\section{Introduction}

The human cornea consists of 5 recognized layers: the epithelium, stroma, endothelium, Bowman layer, and Descemet membrane. It is a transparent hydrogel and having elastic barrier for protecting the intraocular tissues[1]. The corneal stroma provides the bulk of the structural framework of the cornea and comprises approximately $80 \%$ to $90 \%$ of its thickness[2] .Mostly the stroma comprises of about 200 orthogonally oriented lamellae, and each of them is made of long uniform collagen fibrils lying parallel one to another with regular inter-fibril spacing[3,4].

Vision loss results from corneal diseases, which are the second cause of blindness[1]. The corneal blindness is well known to accounts for nearly 10 million cases of vision loss worldwide and $\sim 40,000$ corneal transplants are performed in United States of America alone[5]. It is a serious problem of public health, social and economy. To address the growing need for corneal transplants two main approaches are being pursued: allogenic and synthetic material. The use of allogenic materials suffers from a number of disadvantages. The major drawback is limited to the availability of quality donor graft materials[6,7]. The short supply and conditions that are not amenable to donor grafting demand further efforts to develop corneal substitutes[1,8].]

The appropriate mechanical properties and excellent optical performance of the corneal substitutes are required for corneal tissue regeneration. In addition, the good biocompatibility for cell proliferation and organization of the extracellular matrix of the substitutes are also needed.9 Several investigations on the corneal tissue repairs are carried out continuously.

Silk fibroin biomaterial shows potential application in corneal substitues[10,11]. Silk is used as a suture for centuries, therefore, silk as a biomaterial for cornea and other tissue regenerations become attractive preposition for tissue engineering application[12]. Silk protein fibroin is obtained from the cocoon of the mulberry silkworm Bombyx mori. It contains two main types of proteins: fibroin and sericin[13]. Fibroin, makes up about $75 \%$ of the protein content and another silk protein called sericin (glue protein) contains rest of silk cocoon. Fibroin is an insoluble protein composed of up to $90 \%$ glycine, alanine and serine. There are heavy chain $(392 \mathrm{KDa})$ and a light chain $(25 \mathrm{KDa})[14,15]$. The silk fibroin is reported that it has non-immunogenic response upon in vivo 
implantation, controllable degradation rate, excellent mechanical properties and good oxygen/water vapor permeability[16,17].

The carbamate can be produced by many methods and it is easily available like silk cocoons. Carbamate is used in different fields such as pesticide, textile finish and surface active agent $[18,19]$. In the field of medicine the carbamate is used as sedative for a long time. The carbamate is also applied as antipyotic, muscle relaxant, analgesic and antiepileptic dugs[20]. The carbamic acid ester shows the function of regulating the central nervous system and is an anticancer agent[21]. The carbamate proves to be potential material in medicine. Blending with silk fibroin biomaterial is a good choice for making matrices for cell growth.

Fibroin films, which are easily dissloved in water[22], are weak both in strength and breaking strain[23]. Methyl carbamate or ethyl carbamate are small molecules with strong hydrophilic. In this work, to improve their properties, the blend films are fabricated by mixing silk protein fibroin with methyl carbamate or ethyl carbamate in different ratios, respectively. The different biophysical and cell culture properties of the blends are tested. The results indicate that the blend films may be potential for corneal stroma cell regeneration.

\section{Materials and Methods}

\section{Preparation of Silk Protein Fibroin Solution}

Fibroin solution was prepared from raw mulberry fresh silk cocoons of Bombyx mori (Soho Biotechnology Co., Ltd., China).. In order to remove silk protein sericin adhered to the silk fibroin (thread) surface, the cut raw mulberry silk cocoons were boiled in $0.05 \mathrm{wt} \%$ sodium carbonate solution at $100 \mathrm{oC}$ for $30 \mathrm{~min}$. They were washed with distilled water to remove sericin. This procedure was repeated three times followed by drying. For obtaining silk fibroin solution (approximately 3.5\% wt/wt) the dried silk fibroin was dissolved in $9.3 \mathrm{M} \mathrm{LiBr}$ solution at $60 \mathrm{oC}$ for 1h. The solution is dialyzed using dialysis tube for 3 days to remove $\mathrm{LiBr}$ solution completely with several changes of distilled water and with continuous stirring. The collected silk fibroin solution was filtered by medical absorbent cotton. Finally, the concentration of the silk fibroin solution was determined by gravimetric analysis. The pure silk fibroin solution was stored at $4 \mathrm{oC}$ immediately for further use.

\section{Preparation of the Carbamate/Silk Fibroin (SF) Blend Films}

The concentration of the methyl carbamate solution and ethyl carbamate solution were calculated to be $10 \mathrm{wt} \%$. The obtained solution were mixed to get blend solution having designed carbamate to fibroin at weight ratios of $0: 10,1: 10,2: 10,3: 10,4: 10,5: 10$ and $6: 10$. The blend solution was poured into a plastic dish and placed in an air conditioned room at temperature $25 \mathrm{oC} \pm 2 \mathrm{oC}$ and humidity $65 \%$ for $24 \mathrm{~h}$. In addition, the blend films were equilibrated under a constant temperature and humidity for $24 \mathrm{~h}$. Finally, due to the modified treatment by mixing of the carbamate, the transparent water-insoluble blend films were obtained for further experiment.

\section{Structure of the Carbamate/SF Blend Films}

The crystallization pattern of the films was recorded on an automatic X'PERT PRO MPD-ray diffractometer (XRD) instrument. XRD date was collected for $2 \theta$ ranging from $5^{\circ}$ to $45^{\circ}$. Moreover, other parameters taken into consideration were tube current $(35 \mathrm{~mA})$, voltage $(40 \mathrm{Kv})$ and scan speed $(10 \% \mathrm{~min})$.

\section{Dissolution Loss Rate of the Carbamate/SF Blend Films}

First, moisture content (MC) of the blend films was determined by the following method. Briefly, the samples were cut into pieces and conditioned under standard conditions $(20 \mathrm{oC}, 65 \% \mathrm{RH})$ for 24 $\mathrm{h}$ before weighing (W0). The same samples were then dried in an oven at $105 \mathrm{oC}$ for $4 \mathrm{~h}$ before weighing (W1).The moisture content was an average of three different determinations. This was calculated as Eq.(1): 


$$
\mathrm{MC}=\frac{W 0-W 1}{W 0} * 100 \%
$$

Where $\mathrm{W} 0$ and $\mathrm{W} 1$ are the conditioned and dried weight of the films respectively.

The conditioned films (M1) were put into tubes. The distilled water was added to a bath in the ratio of 1:100. The contents were incubated in a water-bathing constant-temperature vibrator at $37 \mathrm{oC}$ for $24 \mathrm{~h}$. The absorbance (A) of the supernatant liquid was measured by an ultraviolet spectrophotometer at $278 \mathrm{~nm}$. The dissolution loss rate (D) of the blend films was an average of three different samples and determined using the following Eq.(2):

$$
\mathrm{D}=\frac{K^{*} A^{*} V}{M 1-M 1 * M C} * 100 \%
$$

Where $\mathrm{K}$ and $\mathrm{V}$ represent the ultraviolet absorption light constant of the SF solution $(\mathrm{K}=1.10)$ and the volume of the solution $(\mathrm{ml})$, respectively.

\section{Light Transmittance Test}

The large carbamate/SF blend films were cut into pieces and placed at the bottom of 24-well plates. The transmittance was measured by a Multiscan spectrophotometer (BIO-TEK SYNERGY). The parameters taken into considerations were the absorbance (A) at $492 \mathrm{~nm}, 550 \mathrm{~nm}$ and $700 \mathrm{~nm}$. The film absorbance was recorded four times and calculated the average. For the purpose of actual application, the transmittance in wet state was tested after the blend films were soaked in distilled water for $24 \mathrm{~h}$ at room temperature. The surface water was wiped off by water absorbing paper. The transmittance was calculated according to the following Eq. (3):

$$
\mathrm{T}=1 / 10 \mathrm{~A} \times 100 \% \text {. }
$$

\section{Mechanical Properties of the Blend Films}

Tensile strength and elongation at break of specimens were determined using a US Instron 3365 testing machine. According to the GB/T1040-2006 protocol the test samples were cut into dumbbell shaped (gauge length $=25.4 \mathrm{~mm}$, width narrow parallel portion $=5 \mathrm{~mm}$, thickness $=$ approximately 40-60 $\mu \mathrm{m}$ ) with a type $3 \mathrm{knife}$ and measured with a tensile speed of $20 \mathrm{~mm} / \mathrm{mine}$ and a clip distance of $28 \mathrm{~mm}$. Tensile strength and elongation in wet state were tested after the blend films soaked in distilled water for $24 \mathrm{~h}$ at room temperature and the surface water was removed by water absorbing paper. The above measurements were repeated at least five times with the same specimen, and the data presented were average value \pm standard deviation.

\section{Cell Culture}

The rabbit corneal stromal cells at 4 to 6 generations were cultured in Dulbecco's modified eagle's medium /Ham's F-12 50/50 Mix(DMEM/F-12 medium, Coring, USA) supplemented with $10 \%$ fetal bovine serum (Biological Industries ) and 1\% UI $/ \mathrm{ml}$ streptomycin-penicillin (Sigma, St Louis, USA) incubated at $37 \mathrm{oC}$ and $5 \% \mathrm{CO} 2$. The Ethyl carbamate/SF blend solution $(3: 10 \mathrm{wt} / \mathrm{wt})$ was poured in 96-well plates (80ul/well)/24-well plates (100ul/well) and spread uniformly at the bottom of well plates, then dried in an air conditioned room at temperature $25 \mathrm{oC}$, humidity $65 \%$ for 24h. The dried blend films were sterilized by gamma irradiation. All the films were submerged in culture medium for $4 \mathrm{~h}$ before cell seeding. Each film was seeded with $1 \times 105$ cells in 24 -well plate and $2 \times 104$ calls in 96-well plate. The culture medium was changed every other day. The fluorescence values at different times (1, 3, 5 and 7 days) were measured using a Multiscan Spectrophotometer (BIO-TEK SYNERGY) by adding Alamar blue to the silk films that were pre-cast in 96-well plates with 4,000 cells per well in $0.2 \mathrm{~mL}$ of complete medium. The cell morphology was observed under inverted fluorescent microscope. 


\section{Results and Discussion}

\section{The Structure of the Carbamate/Silk Fibroin (SF) Blend Films}

Silk fibroin (SF) has two kinds of crystalline modifications, silk I and silk II, as well as the random coil form. In XRD spectra of silk fibroin, the main diffraction peaks of Silk I appeared at $12.2^{\circ}(\mathrm{ms}), 19.7^{\circ}(\mathrm{s}), 24.7^{\circ}(\mathrm{ms}), 28.2^{\circ}(\mathrm{ms})$ and those of Silk II present on $9.1^{\circ}(\mathrm{ms}), 18.9^{\circ}(\mathrm{ms})$ and $20.7^{\circ}$ ( $\mathrm{v} \mathrm{s}$ )[24]. The pure silk fibroin has random coil (amorphous) structure[25]. In order to investigate the crystalline structure of the blend films, XRD of prepared films was carried out and the acquired patterns are shown at Fig. $1 \mathrm{~A}$ and B. When the ratios of carbamate/SF were 1/10 and $2 / 10$ the main diffraction peaks appeared at about $12.2^{\circ}, 19.7^{\circ}$ and $24.3^{\circ}$. It is observed that the main crystalline modification of the blend films was in Silk I and little in Silk II. Silk I crystal was decreased when the content of carbamate was 3/10. Moreover the total crystallinity of the blend films decreased significantly. When the proportion of carbamate/SF were $4 / 10,5 / 10,6 / 10$, the diffraction peaks of silk II crystalline appeared at $9.1^{\circ}, 20.7^{\circ}$ and $24.3^{\circ}$ due to increase in carbamate. Silk I crystal disappeared completely when the content of carbamate was over $3 / 10$ in the blend films.

The presence of carbamate caused structural changes of SF film from random coil to crystalline structure. This decreased the crystallinity of SF and restrained silk I. The carbamate molecules may form H-bonds with silk fibroin and this possibly increased the distance between the silk fibroin chains. The numbers of H-bonds formed between them were decreased[26,27]. Therefore, it was difficult to form the regular crystal structure by H-bonds and became silk I or silk II crystal for silk fibroin molecules. Because of crystallinity the water molecules were inhibited to permit into the films. This caused the carbamate/SF blend films water-insoluble[28].
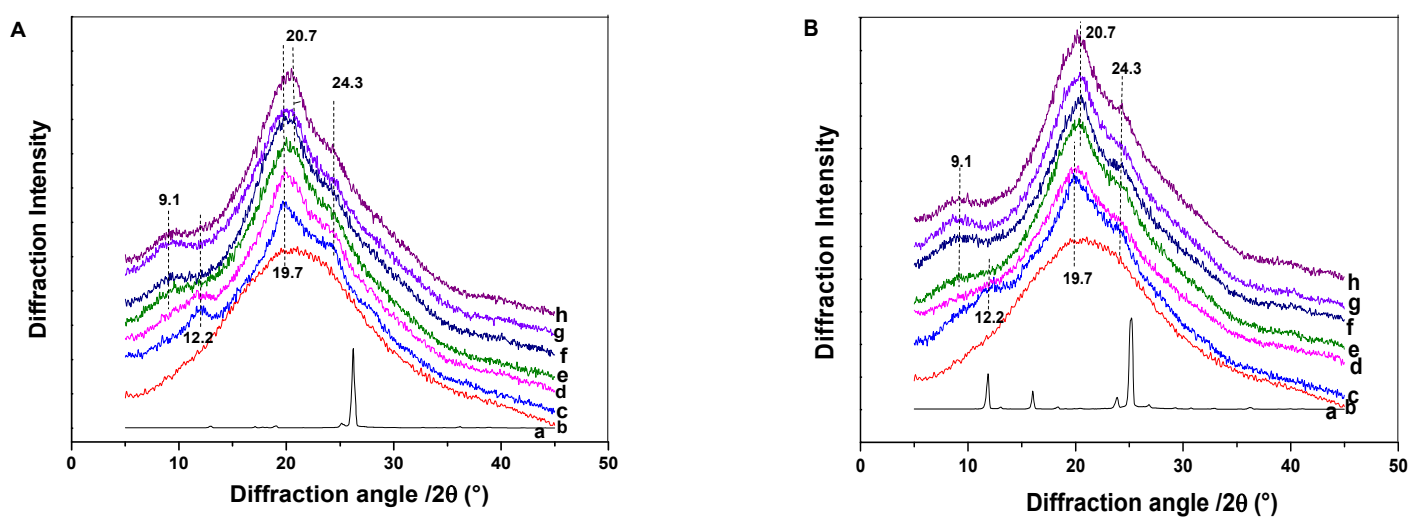

Figure 1(A and B). X-ray diffraction curves of carbamate/silk fibroin (SF) blend films

(A) methyl carbamate/SF; (B) ethyl carbamate/SF

(a) carbamate; (b)-(h): carbamate/SF=0/10 6/10

\section{Dissolution Rate of the Carbamate/SF Blend Films}

It was reported that the pure films were dissolved in water, which limited their applications in vivo[29]. The presence of carbamate changed the structure of the SF films and improved the properties of the SF films. The presence of carbamate modified the water-soluble SF films into water-insoluble blend films. Methyl carbamate and ethyl carbamate show similar phenomenon, which can be observed from Fig.2. The dissolution rate decreased as a whole due to increase in the amount of carbamate. The ratio of the carbamate/SF over $2 / 10$ showed that the silk fibroin dissolution rate was under 5\%. From a general point of view, the biological use of silk films were limited because of their bad water stability[22,30]. This kind of blend films may be helpful for tissue regeneration and tissue engineering application. 

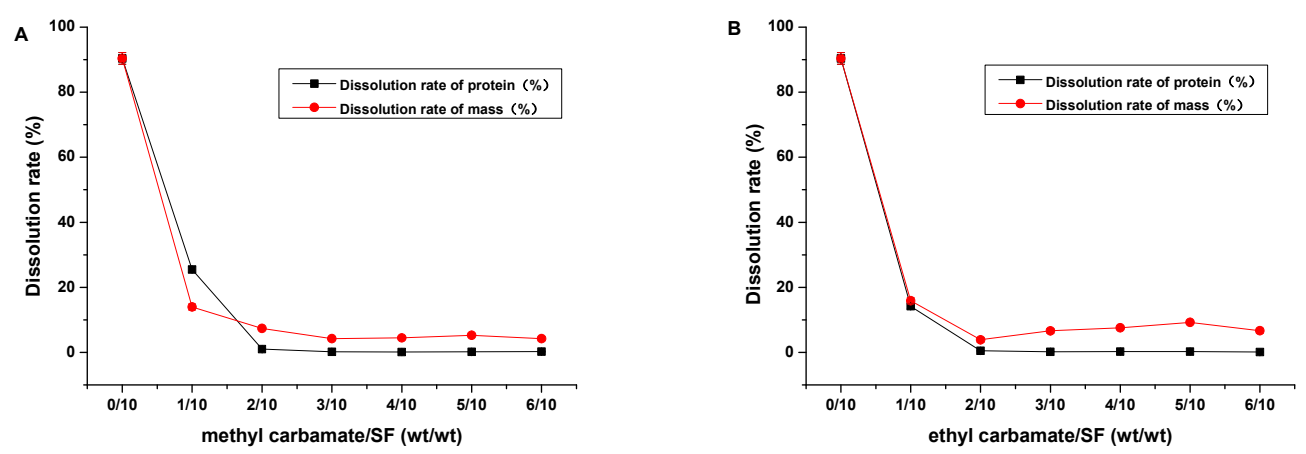

Figure 2 Dissolution rate of carbamate/SF blend films.

(A) methyl carbamate/SF; (B) ethyl carbamate/SF

\section{Light Transparency of Blend Films}

The variation trend of light transmission between 400 and $700 \mathrm{~nm}$ was similar to that of the human cornea. The wavelengths were at $492 \mathrm{~nm}, 550 \mathrm{~nm}$. In order to evaluate the transmittance of the blend films the wavelengths between $550 \mathrm{~nm}$ and $700 \mathrm{~nm}$ were taken into consideration. In dry state the light transmission values of all blend films were increased with the increased of the wavelength and all the values were over $88 \%$. Overall, the transmittance of the blend films in wet state was superior to that of dry state. As showed in Fig. $3 \mathrm{~A}$ and B, the transmittance of blend films in wet state was over $90 \%$. This also exhibited optical stability when the ratio over $2 / 10$, which might be attributed to the stability of the interspace structure of the fibroin molecules with the addition of carbamate. Although there were lot of crystalline domains in the blend films, the crystal grain size was much less than the wavelength of visible light. This did not cause diffraction or scattering of light. A suitable cornea material requires a long-term transparency. In order to obtain the best visual acuity the corneal must maintain transparent and efficient through the incident and reduce scattering[31]. The stability of light transmission of the blend films may be considered as a potential corneal cell material.
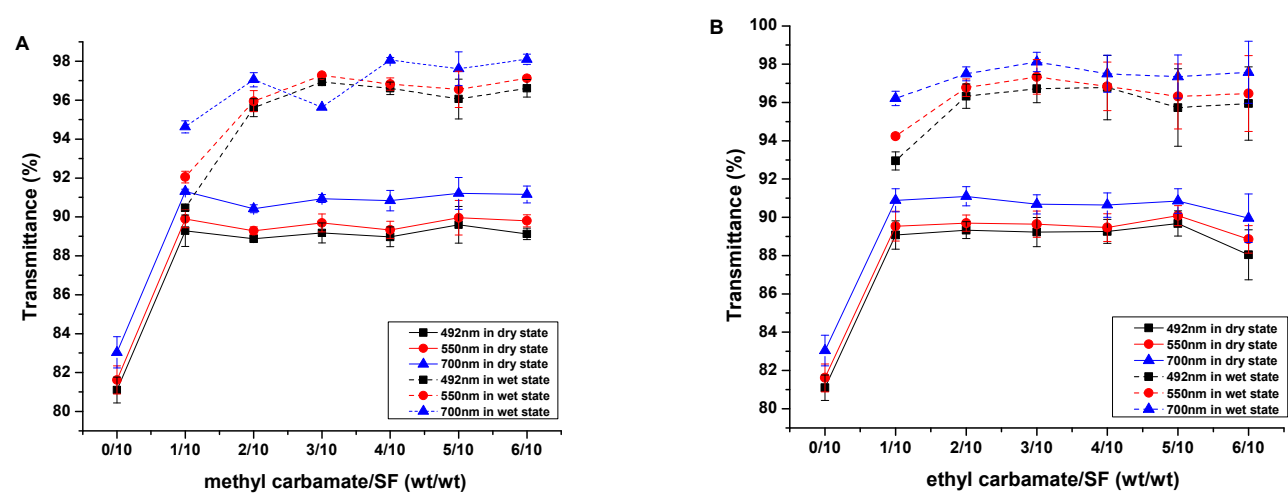

Figure 3.The light transparency of silk films

(A) methyl carbamate/SF blend films (B) ethyl carbamate/SF blend films

\section{Mechanical Properties of Blend Films}

Mechanical properties are also the important factors for assessing the application of the blend films. Tensile test was carried out to evaluate one of the mechanical properties of carbamate/SF blend films both in dry and wet states. All results are presented in Figure 4 (A, B, C and D). Compared with silk film in dry state, both breaking strength and tensile strength of the 
carbamate/SF with a mass ratio of $1 / 10$ decreased. The additive carbamate weakened the interaction forces of silk fibroin (Fig. 4 A and C). Overall, with the increase of carbamate content from 1/10 to $6 / 10$ in dry state, the tensile strength and tensile strength of films increased. When methyl carbamate/SF with a ratio of $6 / 10$, the tensile strength and tensile strength reached their maximum; while when ethyl carbamate/SF with a ratio of $5 / 10$, its tensile strength and tensile strength reached their maximum. The result indicated that carbamate and fibroin formed a typical miscible multi-component materials, and showed high copatibility with XRD results.
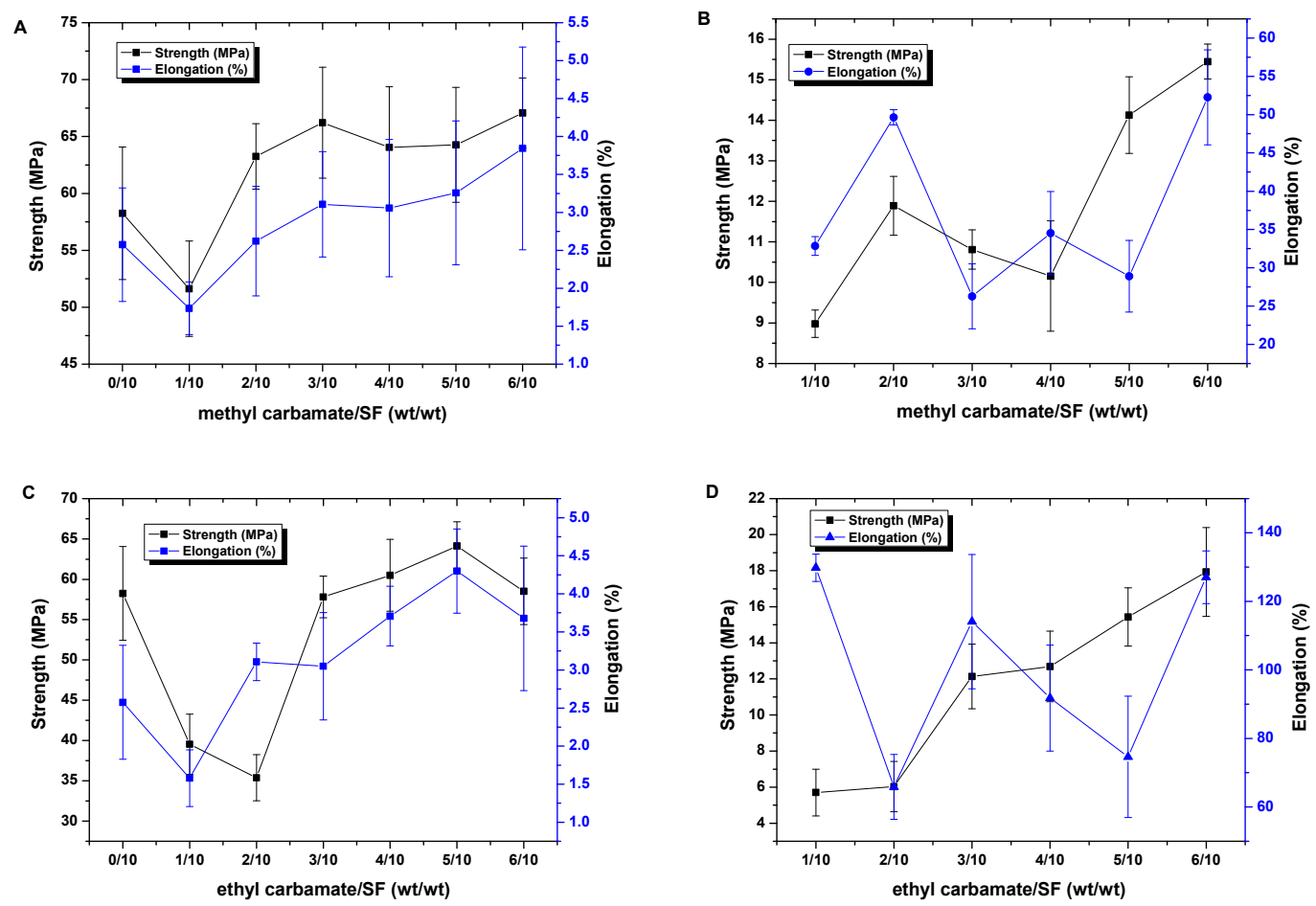

Figure 4. The mechanical properties of silk films.

(A) methyl carbamate/SF blend films in dry state; (B) methyl carbamate/SF blend films in wet state; carbamate/SF blend films in dry state and

(D) ethyl carbamate/SF blend films in wet state

(C) ethyl

As the mechanical properties of films in wet state are essential for its biomedical application. All films were soaked in $0.9 \mathrm{wt} \%$ saline for 24 hours. The elongation of the films in wet state increased significantly, and the broken tensile strength was decreased (Fig. 4 B and D). Water molecules penetration can accelerate motions of unfettered molecular chain segments, which lead to the change of broken strength and elongation in wet state[32]. The films were more flexible by soaking, and much better than previous study[33]. The strength and the elongation of the blend films were reached maximum when methyl carbamate accounts to $2 / 10$, approximately $17.4 \mathrm{MPa}$ and $174 \%$ respectively. The strength and the elongation of the blend films were reached maximum when ethyl carbamate accounts to 6/10, approximate $17.9 \mathrm{MPa}$ and $127 \%$ respectively. The breaking strength and elongation of the human cornea are $3.81 \pm 0.41 \mathrm{MPa}[34]$. The mechacial properities of blend films are still meet the requirments in wet state. It makes the blend films are potential for application in biomedical field in terms of mechanical aspect.

\section{In Vitro Cell Culture and Morphology on the Blend Films}

In order to evaluate the cytocompatibility of the obtained blend material of SF and carbamate, the corneal stromal cell proliferation was observed. After comparing the dissolution rate, optical, and mechanical properties among different ratios, the ratio of $3 / 10$ was chosen to conduct cell culture experiment and the 96-well tissue culture plate as a control group. Figure 5 shows the proliferation result obtained by Alamar blue staining at 1, 3,5 and 7 days after seeding on blend films and culture 
plate (control). The fluorescence value of the cells seeded on the material and the plate surpassed 1000 and showed no difference between them after one day of the seeding. Both groups were proliferated up to confluent stage with the time. On day 3 the cells on the Ethyl carbamate/SF blend films were proliferated faster. The florescence values were increased from 3000 to 6000 approximately. On day 7 not much change is observed. This may be due to the growth periodicity of the cells turned into stationary phase. The blend material showed cytocompatibility. This indicates that the ethyl carbamate/SF blend film may offer a new option for corneal tissue regeneration.

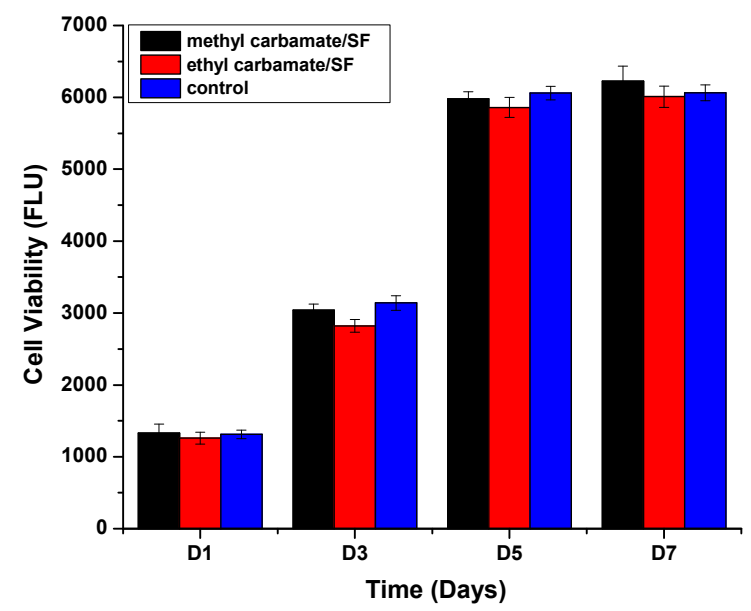

Figure 6. The activity of the corneal stromal cell grown on the blend films $\quad S F /$ carbamate $=3 / 10$.

\section{Conclusion}

The carbamate and silk fibroin blend films are fabricated. The blend films show good optical transparency, better physical properties in dry or wet state. An appropriate protein dissolution loss rate, and cytocompatibility of the blends are observed. The keratinocytes are grown on the films. The results indicate that the silk blends have a good potential for regeneration of corneal stroma cells.

\section{Acknowledgement}

The work is supported by National Natural Science Foundation of China (Grant No. 51373114), PAPD and College Nature Science Research Project of Jiangsu Province, China (Grant No. 15KJA540001).

\section{References}

[1] Polisetti N, Islam M M, Griffith M, Methods in Molecular Biology, J. The Artificial Cornea. 1014 (2013) 45-52.

[2] Delmonte D W, Kim T, Anatomy and physiology of the cornea, J. Journal of Cataract \& Refractive Surgery. 37 (2011) 588-98.

[3] Maurice D M, The structure and transparency of the cornea, J. The Journal of physiology. 136 (1957) 263-286.

[4] Freegard T J, The physical basis of transparency of the normal cornea, J. Eye. 11 (1997) 465-471.

[5] Upadhyay M P, J. Corneal blindness : a global perspective. (2001).

[6] Ghezzi C E, Rnjak-Kovacina J, Kaplan D L, Corneal tissue engineering: recent advances and 
future perspectives, J. Tissue Engineering Part B: Reviews. 21 (2015) 278-287.

[7] Tan D T H, Dart J K G, Holland E J, et al, Corneal transplantation, J. The Lancet. 379 (2012) 1749-1761.

[8] Chandra S R, Global blindness: VISION 2020: the right to sight, J. Archives of Ophthalmology. 126 (2008) 1457-1457.

[9] Liu Y, Ren L, Long K, et al, Preparation and characterization of a novel tobramycin-containing antibacterial collagen film for corneal tissue engineering, J. Acta Biomaterialia. 10(2014) 289-99.

[10] Chen J, Yan C, Zhu M, et al, Electrospun nanofibrous SF/P(LLA-CL) membrane: a potential substratum for endothelial keratoplasty, J. International Journal of Nanomedicine. 10 (2015) 3337-3350.

[11] Liang Jia , Chiara E. Ghezzi , Kaplan D L, Optimization of silk films as substrate for functional corneal epithelium growth, J. Journal of Biomedical Materials Research Part B Applied Biomaterials. (2015).

[12] Moy R L, Lee A, Zalka A, Commonly used suture materials in skin surgery, J. Am Fam Physician. 44 (1991) 2123-2128.

[13] Kato N, Sato S, Yamanaka A, et al, Silk protein, sericin, inhibits lipid peroxidation and tyrosinaseactivity. J. Bioscience, biotechnology, and biochemistry. 62 (1998) 145-147.

[14] Phillips D M, Drummy L F, Conrady D G, et al, Dissolution and regeneration of Bombyx mori silk fibroin using ionic liquids, J. Journal of the American Chemical Society. 126 (2004) .

[15] Ming J, Zuo B, Silk I structure formation through silk fibroin self-assembly, J. Journal of Applied Polymer Science. 125 (2012) 2148-2154.

[16] Vepari C, Kaplan D L, Silk as a biomaterial, J. Progress in polymer science. 32 (2007) 991-1007.

[17] Meinel L, Hofmann S, Karageorgiou V, et al, The inflammatory responses to silk films in vitro and in vivo, J. Biomaterials. 26 (2005) 147-155.

[18] And J B, Patterson H H, Photodecomposition of the Carbamate Pesticide Carbofuran: Kinetics and the Influence of Dissolved Organic Matter, J. Environmental Science \& Technology. 33 (1999) 874-881.

[19] Bak A, Reinhardt R M, Reaction equilibria and textile performance from low formaldehyde release carbamate finishing, J. Textile Research Journal. 59 (1989) 53-60.

[20] Emig W, Aponal in the therapy of organic coronary blood flow disorders, J. Deutsches Medizinisches Journal. 21 (1970) 816-22.

[21] Nettleship A, Henshaw P S, Meyer H L, Induction of Pulmonary Tumors in Mice With Ethyl Carbamate (Urethane), J. JNCI: Journal of the National Cancer Institute. 52 (1943) 290-295.

[22] Hu X, Shmelev K, Sun L, et al, Regulation of silk material structure by temperature-controlled water vapor annealing, J. Biomacromolecules. 12 (2011) 1686-96.

[23] Giuliano F, Masuhiro T, Silvia B, Structure and physical properties of silk fibroin/ polyacrylamide blend films, J. Journal of Applied Polymer Science. 71 (1999) 1563-1571.

[24] Lu Q, Hu X, Wang X, et al, Water-insoluble silk films with silk I structure, J. Actabiomaterialia. 6 (2010) 1380-1387.

[25] Meinel L, Hofmann S, Karageorgiou V, et al, The inflammatory responses to silk films in vitro and in vivo, J. Biomaterials. 26 (2005) 147-155.

[26] Marsh R E, Corey R B, Pauling L, An investigation of the structure of silk fibroin, J. Biochimica Et Biophysica Acta. 16 (1955) 1-34. 
[27] Bourne P E, Weissig H, Structural Bioinformatics, Volume 44, J. Methods of Biochemical Analysis. 44 (2003) 321-337.

[28] Numata K, Cebe P, Kaplan D L, Mechanism of Enzymatic Degradation of Beta-sheet Crystals, J. Biomaterials.31 (2010) 2926-33.

[29] Zhang S, Li J, Yin Z, et al, Silk fibroin composite membranes for application in corneal regeneration, J. Journal of Applied Polymer Science. 132 (2015).

[30] Kweon H, Ha H C, Um I C, et al, Physical properties of silk fibroin/chitosan blend films, J. Journal of Applied Polymer Science. 80 (2001) 928-934.

[31] Qazi Y, Wong G, Monson B, et al, Corneal transparency: genesis, maintenance and dysfunction, J. Brain research bulletin. 81 (2010) 198-210.

[32] Shang S, Zhu L, Fan J, Physical properties of silk fibroin/cellulose blend films regenerated from the hydrophilic ionic liquid, J. Carbohydrate Polymers. 86 (2011) 462-468.

[33] Liang Zhou, Qin Wang, Jianchuan Wen, et al, Preparation and characterization of transparent silk fibroin/cellulose blend films, The political transformation of Spain after Franco /. Praeger, 1979.

[34] Zeng Y, Yang J, Huang K, et al, A comparison of biomechanical properties between human and porcine cornea, J. Journal of biomechanics. 34 (2001) 533-537. 